\title{
The Effect of Sodium Alginate Supplementation with HIIT or MICT on Glucagon and Glucagon-Like Peptide- 1 in Women with Metabolic Syndrome
}

\section{Rezvan Azizi}

Razi University of Kermanshah: Razi University

Mohammad Azizi ( $\sim$ azizimihammad@gmail.com )

Razi University https://orcid.org/0000-0002-6386-7344

Worya Tahmasebi

Razi University of Kermanshah: Razi University

Hadi Abdollhzad

Kermanshah University of Medical Sciences

\section{Research Article}

Keywords: Metabolic Syndrome, high intensity interval training, moderate-intensity continuous training, glucagon, glucagon-like peptide-1

Posted Date: December 13th, 2021

DOI: https://doi.org/10.21203/rs.3.rs-1127688/v1

License: (c) (1) This work is licensed under a Creative Commons Attribution 4.0 International License. Read Full License 


\section{Abstract}

Background: Exercise intervention and dietary supplementation are considered as approaches to manage obesity, as well as, metabolic syndrome (MetS). The aim of the present study was to evaluate the effect of sodium alginate supplementation with high intensity interval training (HIIT) or moderate-intensity continuous training (MICT) on serum levels of glucagon and glucagon-like peptide-1 (GLP-1) in women with MetS.

Methods: This randomized controlled clinical trial was performed on 46 women with MetS. Participants were randomly divided to six groups including: 1) HIIT+ sodium alginate (HS, n=8);2) HIIT+ placebo (HP, $n=8) ; 3) M I C T+$ sodium alginate $(M S, n=8) ; 4)$ MICT+ placebo $(M P, n=8) ; 5)$ sodium alginate $(S, n=7)$; and 6$)$ control group $(P, n=7)$. Sodium alginate were prescribed 1.5 gram alone or along with HIIT or MICT sessions (3 times per week). Anthropometric indices, glycemic profile, serum level of glucagon and GLP-1 were measured before and after 8 weeks intervention.

Results: In HS, HP, MS, and MP groups the mean of weight $(p=0.000)$, body mass index $(p=0.001)$, fasting blood sugar $(p=0.003)$, insulin $(p=0.000)$, insulin resistance $(p=0.043)$, and glucagon $(p=0.011)$ decreased significantly, however GLP_1 $(p=0 / 01)$ level increased significantly after intervention.

Conclusion: Since HIIT and MICT with or without sodium alginate supplementation leads to improvement in anthropometric indices, glycemic profile, serum GLP-1 and glucagon levels, it can be stated that the training method of the present study is suitable for improving and reducing complications associated with MetS in women.

\section{Trial registration: IRCT20191116045457N1. Registered 27 jan 2020, https://en.irct.ir/trial/43776}

\section{Introduction}

Obesity is considered as the important public health challenge that the health system in most countries is involved in its related complications(1). This phenomenon is associated with many diseases and disorders of the heart, diabetes, as well as some cancers, hypertension, metabolic syndrome (MetS), and osteoarthritis, which have led to obesity was remembered as one of the most serious threats to human health (2).

MetS refers to the presence of at least three indicators of five risk components, including triglyceride more than 150 $\mathrm{mg} / \mathrm{dl}$, high density lipoprotein (HDL) less than $52 \mathrm{mg} / \mathrm{dl}$, systolic blood pressure (SBP) greater than $130 \mathrm{mmHg}$ or diastolic blood pressure (DBP) greater than $85 \mathrm{mmHg}$, fasting blood sugar (FBS) higher than $100 \mathrm{mg} / \mathrm{dl}$, and waist circumference (WC) more than $88 \mathrm{~cm}$ in women. This syndrome is not a disease in itself, but a set of undesirable features associated with bad habits and lifestyle, and obesity is strongly associated with an increased prevalence (3).

Factors that contribute to management of obesity and overweight include lifestyle modification, regular physical activity, dietary intervention, and psychological factors. Lack of physical activity and excess calorie intake is associated with obesity, which is associated with increased plasma free fatty acids. An imbalance between free fatty acid intake and fatty acid oxidation leads to the accumulation of lipids in the body and consequently overweight and obesity (4).

Physical activity as high intensity interval training (HIIT) has been recommended for people with limited time to achieve health (5). On the other hand, moderate intensity continuous training (MICT) can help control and improve the weight loss process due to the volume of adjusted training and less impact (6). Another method to deal with the 
problems caused by obesity is herbal supplementation in which can be combined with regular physical activity to lose more weight, as well as, contribute to attenuate MetS, type 2 diabetes, and cardiovascular diseases (7). In this regard, the use of regular physical activity with herbal supplements is one of the proposed methods to lose weight and appetite management. Accordingly, in the present study, the effect of sodium alginate supplementation with HIIT or MICT was investigated on serum levels of glucagon and glucagon-like peptide-1 (GLP-1) in women with MetS.

\section{Method}

Study design and participants

This randomized clinical trial was performed on women with MetS. This trial was registered in the Iranian Clinical Trial Registration Center (IRCT20191116045457N1) and was approved by the Ethics Committee of Kermanshah University of Medical Sciences (IR.KUMS.REC.1398.453) and. After explaining the study aims, an informed consent form was completed and signed by all studied subjects.

Eligible subjects who had MetS were 20-30 years old women with body mass index (BMI) 25-35 kg/ m². MetS was diagnosed based on the National Cholesterol Education Program (NCEP) Adult Treatment Panel III (ATP III), which was defined the presence at least of three of the five following factors:

$-\mathrm{TG}>150 \mathrm{mg} / \mathrm{dl}, \mathrm{HDL}<52 \mathrm{mg} / \mathrm{dl}, \mathrm{SBP}>130 \mathrm{~mm} \mathrm{Hg}$ or $\mathrm{DBP}>85 \mathrm{~mm} \mathrm{Hg}, \mathrm{FBS}>100 \mathrm{mg} / \mathrm{dl}$, and WC $>88 \mathrm{~cm}$.

None of the subjects had a history of regular physical activity in the past year. In addition, they were asked to refrain from any sports activities and do not change their diet. After applying the inclusion and exclusion criteria, 46 women with MetS were entered the study and randomly divided to six groups including: 1) HIIT+ sodium alginate (HS, $n=8)$; 2) HIIT+ placebo (HP, $n=8) ; 3)$ MICT+ sodium alginate (MS, $n=8) ; 4) M I C T+$ placebo $(M P, n=8) ; 5)$ sodium alginate ( $($, $n=7)$; and 6) control group $(P, n=7)$.

Anthropometry measurement

To measure the weight of the subjects, the digital scale (Camry, model EB9003, Japan) was used with an accuracy of $0.1 \mathrm{~kg}$ while participants had the least clothing and without shoes. Height was measured using a Seca stadiometer (Seca scale, Germany) with an accuracy of $0.1 \mathrm{~cm}$ while subjects were in standing position without shoes. A nonstretched tape was used to measure waist circumference (WC) at the top of the iliac crest.

Dietary intake assessment

The dietary intake was evaluated using a 24-hour food recall questionnaire three days per week at the beginning and last week of intervention. The food items was assessed by NUTRITIONIST IV software based on the United States Department of Agriculture Food Composition Table that was modified for Iranian foods.

Blood pressure measurement

Blood pressure of subjects was measured by a digital sphygmomanometer (ZYKlusmed BP HS-20A, Japan) in sitting position and on the right hand two times with ten-minutes interval and the average was recorded.

Blood sampling and analysis

Blood sampling was done in two stages including the beginning of the study and 48 hours after the last training session. In addition, subjects were asked not to do any physical activity for 48 hours prior to blood sampling. At each 
time, $10 \mathrm{ml}$ of fasting blood venous (12 hours) was obtained from the subjects. Blood samples were centrifuged and obtained serum were stored at $-80^{\circ} \mathrm{C}$ until analysis. Fasting blood sugar (FBS) was evaluated by glucose oxidase method (Pars Azmon Co, Tehran, Iran). Lipid profiles including (HDL) and (TG) concentration were measured by enzymatic kits (Pars Azmon Co, Tehran, Iran).

Intervention

The training and supplementation period was lasted for 8 weeks including 3 training sessions per week and the supplement was taken only on training days (30 minutes before training sessions). Three groups including HS, MS, and $\mathrm{S}$ groups consumed $1.5 \mathrm{~g}$ of sodium alginate (Sigma, USA) solution in $100 \mathrm{mg}$ of water(9).

The training protocol also included 30 minutes of HIIT on a treadmill (HP cosmed pulsar, Germany) for 4 minutes at high intensity [85 to $95 \%$ heart rate (HR) max] and with rest periods for 3 minutes with intensity [50\% HR max] 4 times. According to the principle of overload, the training in the first and second weeks started with 65 and $75 \% \mathrm{HR}$ max, respectively, and in the third and fourth weeks reached 85 and 90\% HR max, respectively (10).

MICT sessions were performed after 10 minutes of warm-up for 30 minutes with $60-70 \%$ reserve HR, and Borg pressure perception scale 11-13 in the first two weeks with an intensity of $60 \%$ and Borg pressure perception scale 11 , in the second two weeks with an intensity of $65 \%$ reserve HR and Borg pressure perception scale 12 , and in the last four weeks with an intensity of $70 \%$ reserve HR and Borg pressure perception scale 13 (11). The subjects' HR was monitored by a HR sensor (Beaver model, AS81 Body shape, Germany) during training.

\section{Statistical analysis}

Data were analyzed using SPSS software version 20 (SPSS Inc., Chicago, IL). To determine the normality of data distribution, Shapiro-Wilk test was used. To compare the variables measured in pre-test and post-test, paired t-test was used. Changes between groups were investigated by one way ANOVA, and if significant, LSD post hoc test were used. Significant level for all analyzes $<0.05$ was considered.

\section{Results}

In present trial, we found that the mean of weight, BMI, and WHR were significantly decreased in HS, HP, MS, and MP groups. Basic characteristics of studied women with MetS and anthropometric indices before and after intervention are presented in Table 1. Dietary intake including energy and macronutrients are reported in Table 2. 
Table 1

Basic characteristics of studied women with MetS and anthropometric indices before and after intervention

\begin{tabular}{|c|c|c|c|c|c|c|}
\hline Variables & $\begin{array}{l}\text { HIIT+S } \\
(n=8)\end{array}$ & $\begin{array}{l}\text { MICT+S } \\
(n=8)\end{array}$ & $\begin{array}{l}\text { HIIT } \\
(n=8)\end{array}$ & $\begin{array}{l}\text { MICT } \\
(n=8)\end{array}$ & $\begin{array}{l}\text { Supplement } \\
(n=7)\end{array}$ & $\begin{array}{l}\text { C } \\
(n=7)\end{array}$ \\
\hline Age & $23 \pm 2.7 *$ & $20.5 \pm 1.7$ & $23.8 \pm 3.65$ & $22 \pm 1.85$ & $22.66 \pm 2.78$ & $20.57 \pm 2.13$ \\
\hline Height & $161.89 \pm 1.4$ & $165.75 \pm 7.24$ & $159 \pm 4.81$ & $163.6 \pm 6.62$ & $160.98 \pm 2.16$ & $164.83 \pm 6.4$ \\
\hline \multicolumn{7}{|c|}{ Bodyweight (kg) } \\
\hline Before & $85.88 \pm 9.07$ & $90.65 \pm 13.21$ & $86.3 \pm 6.14$ & $86.9 \pm 8.09$ & $87.14 \pm 8.3$ & $89.56 \pm 8.47$ \\
\hline After & $80.02 \pm 9.66$ & $86.65 \pm 12.73$ & $82.21 \pm 6.77$ & $83.8 \pm 8.5$ & $86.21 \pm 8.29$ & $89.12 \pm 8.41$ \\
\hline P-Value & $<0.0001$ & $<0.0001$ & $<0.0001$ & $<0.0001$ & 0.854 & 0.1 \\
\hline \multicolumn{7}{|c|}{$\mathrm{BMI}\left(\mathrm{kg} / \mathrm{m}^{2}\right)$} \\
\hline Before & $33.13 \pm 2.2$ & $33.29 \pm 3.99$ & $34.13 \pm 3.64$ & $32.71 \pm 0.97$ & $34.03 \pm 1.07$ & $33.3 \pm 2.26$ \\
\hline After & $30.78 \pm 3.8$ & $31.82 \pm 3.61$ & $32.51 \pm 4.24$ & $31.55 \pm 1.36$ & $33.66 \pm 2.78$ & $33.14 \pm 2.51$ \\
\hline P-Value & $<0.0001$ & 0.001 & 0.016 & 0.001 & 0.374 & 0.085 \\
\hline \multicolumn{7}{|l|}{ WC } \\
\hline Before & $135.8 \pm 16.1$ & $139 \pm 11.2$ & $134.5 \pm 22.5$ & $131.9 \pm 24.1$ & $134.8 \pm 17.4$ & 135. $2 \pm 0.4$ \\
\hline After & $133.6 \pm 14.1$ & $133.9 \pm 21.1$ & $132.83 \pm 16.7$ & $130.3 \pm 19.6$ & $133.9 \pm 18.3$ & $134.1 \pm 0.5$ \\
\hline P-Value & 0.029 & 0.023 & 0.041 & 0.032 & 0.325 & 0.21 \\
\hline \multicolumn{7}{|l|}{ Vo2max } \\
\hline Before & $27.77 \pm 1.85$ & $28.62 \pm 2.38$ & $29.4 \pm 1.34$ & $30 \pm 3.16$ & $30.12 \pm 1.48$ & $30.62 \pm 2.92$ \\
\hline After & $31.44 \pm 1.79$ & $31.12 \pm 2.41$ & $31.8 \pm 2.58$ & $31 \pm 2.82$ & $31.11 \pm 1.45$ & $30.27 \pm 2.19$ \\
\hline P-Value & 0.021 & 0.033 & 0.003 & 0.001 & 0.42 & 0.573 \\
\hline \multicolumn{7}{|c|}{ *Mean \pm SD } \\
\hline \multicolumn{7}{|c|}{$\begin{array}{l}\text { BMI: body mass index; WHR: waist to hip ratio; S: supplementation with sodium alginate; HIIT: high intensity } \\
\text { interval training; MICT: moderate-intensity continuous training }\end{array}$} \\
\hline
\end{tabular}


Table 2

Energy and macronutrients before and after intervention

\begin{tabular}{|c|c|c|c|c|c|c|c|c|}
\hline \multirow[t]{2}{*}{ Variables } & & \multirow[t]{2}{*}{ Energy $(\mathrm{Kcal} / \mathrm{d})$} & \multicolumn{2}{|l|}{ Protein } & \multicolumn{2}{|c|}{ Carbohydrates } & \multicolumn{2}{|l|}{ Fat } \\
\hline & & & Kcal/d & $g / d$ & $\mathrm{Kcal} / \mathrm{d}$ & $g / d$ & $\mathrm{Kcal} / \mathrm{d}$ & $\mathrm{g} / \mathrm{d}$ \\
\hline \multirow[t]{2}{*}{$\mathrm{HIIT+S}$} & Before & 3186 & 804.68 & 201.17 & 1608 & 402 & 774 & 86 \\
\hline & After & 3062 & 746.72 & 186.68 & 1560 & 390 & 756 & 84 \\
\hline \multirow[t]{2}{*}{$\mathrm{MICT}+\mathrm{S}$} & Before & 3089 & 794.96 & 198.74 & 1584 & 396 & 711 & 79 \\
\hline & After & 2886 & 742.04 & 185.51 & 1496 & 374 & 648 & 72 \\
\hline \multirow[t]{2}{*}{ HIIT } & Before & 3014 & 668 & 167 & 1680 & 420 & 666 & 74 \\
\hline & After & 2889 & 588.6 & 147.15 & 1644 & 411 & 657 & 73 \\
\hline \multirow[t]{2}{*}{ MICT } & Before & 2959 & 597.28 & 149.32 & 1624 & 406 & 738 & 82 \\
\hline & After & 2901 & 572 & 143 & 1600 & 400 & 729 & 81 \\
\hline \multirow[t]{2}{*}{ Supplement } & Before & 3067 & 717.92 & 179.48 & 1576 & 397 & 774 & 86 \\
\hline & After & 2996 & 700.84 & 175.21 & 1540 & 385 & 756 & 84 \\
\hline \multirow[t]{2}{*}{ Control } & Before & 3166 & 741.41 & 185.41 & 1624 & 406 & 801 & 89 \\
\hline & After & 3065 & 692.68 & 173.17 & 1608 & 402 & 765 & 85 \\
\hline
\end{tabular}

Our findings were indicated that the GLP-1 level after 8 weeks intervention was significantly increased in $\mathrm{HS}(\mathrm{p}=0.000), \mathrm{HP}(\mathrm{P}=0.000) \mathrm{MS}(0.014)$, and $\mathrm{MP}(0.000)$ groups, while in sodium alginate and control group were not seen any difference after intervention. (Table 3) Furthermore, the glucagon hormone level was significantly decreased in in $\mathrm{HS}(\mathrm{p}=0.000), \mathrm{HP}(\mathrm{P}=0.001) \mathrm{MS}(0.016)$, and $\mathrm{MP}(0.032)$ groups. (Table 3$)$ 
Table 3

Comparison of GLP-1 and Glucagon hormones changes before and after 8 weeks intervention within groups

\begin{tabular}{|c|c|c|c|c|c|c|}
\hline Variables & $\begin{array}{l}\text { HIIT+S } \\
(n=8)\end{array}$ & $\begin{array}{l}\text { MICT+S } \\
(n=8)\end{array}$ & $\begin{array}{l}\text { HIIT } \\
(n=8)\end{array}$ & $\begin{array}{l}\text { MICT } \\
(n=8)\end{array}$ & $\begin{array}{l}\text { Supplement } \\
(n=7)\end{array}$ & $\begin{array}{l}\text { C } \\
(n=7)\end{array}$ \\
\hline \multicolumn{7}{|c|}{ GLP-1 (pg/ml) } \\
\hline Before & $186.33 \pm 8.86^{\star}$ & $180.5 \pm 9.47$ & $191.11 \pm 9.22$ & $185.87 \pm 5.84$ & $206.28 \pm 13.53$ & $204 \pm 12.28$ \\
\hline After & $208.22 \pm 8.18$ & $202.62 \pm 13.29$ & $209.55 \pm 7.48$ & $206.5 \pm 6.47$ & $207.4 \pm 8.67$ & $203 \pm 8.86$ \\
\hline P-Value & $<0.0001$ & 0.014 & $<0.0001$ & $<0.0001$ & 0.139 & 0.199 \\
\hline \multicolumn{7}{|c|}{ Glucagon (n/ml) } \\
\hline Before & $379.16 \pm 17.39$ & $351.52 \pm 19.64$ & $328.33 \pm 53.98$ & $372.82 \pm 15$ & $364.9 \pm 14.27$ & $338.7 \pm 51.95$ \\
\hline After & $376.27 \pm 16.93$ & $348.48 \pm 24.52$ & $327.48 \pm 39.25$ & $370.27 \pm 16.11$ & $363.3 \pm 13.15$ & $335.54 \pm 54.22$ \\
\hline P-Value & $<0.0001$ & 0.016 & 0.001 & 0.032 & 0.117 & 0.125 \\
\hline \multicolumn{7}{|c|}{ *Mean \pm SD } \\
\hline \multicolumn{7}{|c|}{$\begin{array}{l}\text { GLP-1: glucagon-like peptide-1; S: supplementation with sodium alginate; HIIT: high intensity interval training; } \\
\text { MICT: moderate-intensity continuous training }\end{array}$} \\
\hline
\end{tabular}

Although, there were no significant deference between level of GLP-1 and glucagon between studied groups in baseline, also after intervention the level of glucagon was not different between groups; however a significant difference was seen in GLP-1 level between studied groups after intervention $(P=0.012)$. (Table 4) 
Table 4

Comparison of differences in GLP-1 and Glucagon hormones changes before and after 8 weeks intervention within and between groups

\begin{tabular}{|c|c|c|c|c|c|}
\hline \multicolumn{2}{|c|}{ Variables } & df & Mean square & $F$ & P-value \\
\hline \multicolumn{6}{|c|}{ GLP-1 (pg/ml) } \\
\hline \multirow[t]{2}{*}{ Before } & Between groups & 5 & 318.46 & 3.56 & 0.109 \\
\hline & Within groups & 40 & 89.35 & & \\
\hline \multirow[t]{2}{*}{ After } & Between groups & 5 & 105.03 & 1.36 & 0.012 \\
\hline & Within groups & 40 & 77.15 & & \\
\hline \multicolumn{6}{|c|}{ Glucagon (n/ml) } \\
\hline \multirow[t]{2}{*}{ Before } & Between groups & 5 & 82.47 & 3.3 & 0.114 \\
\hline & Within groups & 40 & 52.83 & & \\
\hline \multirow[t]{2}{*}{ After } & Between groups & 5 & 97.46 & 3.16 & 0.117 \\
\hline & Within groups & 40 & 82.04 & & \\
\hline \multicolumn{6}{|c|}{ GLP-1: glucagon-like peptide-1 } \\
\hline \multicolumn{6}{|c|}{ P-values were obtained one way ANOVA. } \\
\hline
\end{tabular}

\section{Discussion}

According to the results of the present study, after 8 weeks weight, BMI, WHR decreased significantly and $\mathrm{VO}_{2} \mathrm{max}$ increased significantly in HS, HP, MS, and MP groups. Although in all mentioned four groups was seen a change in anthropometric indices due to exercise, the average of these changes according to Table 1 in the HS is higher than other groups. This indicates the greater impact of HS on anthropometric indices improving.

In this regard, Nikroo et al. (12) showed that both HIIT and MICT were significantly increased the $\mathrm{VO}_{2}$ max and were significantly reduced the BMI. However, they showed a significant difference between the two groups in increasing the $\mathrm{VO}_{2}$ max. Suri et al. (13) showed that 10 weeks of aerobic exercise led to a significant reduction in weight, BMI, and body fat percentage. In another study, Kurdi et al. (14) reported a significant increase in $\mathrm{VO}_{2}$ max, a decrease in body fat percentage, weight, BMI, and WC following 8 weeks of endurance training. In addition, Farazmandi et al. (15) who examined the effect of HIIT on obese women, reported that these exercises significantly increased $\mathrm{VO}_{2}$ max consumption, decreased body fat percentage, weight, BMI and WC. Studie have shown that do exercise significantly reduces subcutaneous fat, especially abdominal fat, as well as $\mathrm{BMI}$, and improves $\mathrm{VO}_{2}$ max consumption and insulin sensitivity (16). Overall, our findings showed that 8 weeks of HIIT or MICT with or without sodium alginate supplementation could improve anthropometric indices, as well as components of MetS(17).

Furthermore, this study showed that GLP-1 hormone was significantly increased in HS, HP, MS, and MP groups. GLP1 the gastric inhibitory polypeptide, is an incretin, which can reduce blood sugar through insulin secretion stimulating and this peptide hormone has several protective and regulatory effects $(16,18)$. Hallworth et al. investigated the effect of continuous exercise on GLP-1 in overweight women and reported that intermittent exercise increases the hormone GLP-1 in overweight women (19). On the other hand, in another study, Martin et al. was showed that the 
intense intermittent and continuous exercise had no significant effect on the level of GLP-1 hormone in overweight subjects after 12 weeks with 3 sessions per week (20). In another study, Islam et al. investigated the effect of HIIT and MICT on GLP-1 and observed that these exercises caused a significant increase in GLP-1(21). Jafari et al. investigated the effect of intermittent aerobic exercise (for 12 weeks) which includes running on a treadmill with periods of four minutes with an intensity of $85-95 \%$ of HR max and three minutes of active rest with an intensity of 65-75\% HR max on Acylated Ghrelin, Peptide Tyrosine Tyrosine and Glucagon-Like Peptide-1 (22). The results showed that the level of GLP-1 remained unchanged in the exercise group compared to the control group after eight and 12 weeks of training (23). In study by Lee et al. (24) was reported the HIIT ( $80 \%$ reserve HR) could increase GLP-1 levels in patients with type 2 diabetes compared to low-intensity interval training (LIIT) (45\% reserve HR). In contrast, the results of Farzanegi study (24) indicated that 8 weeks of aerobic exercise on a treadmill on mice decreased GLP-1 levels. In relation to this inequality, we can point to the differences in subjects and the intensity of training that this study was performed on rats and the results of the present study on glucagon did not show a significant change. Inconsistent studies with the present study showed that glucagon levels increased during acute activity and after recovery (26). Yada et al. (27) reported that following one hour of cycling with $50 \%$ of $\mathrm{VO}_{2}$ max consumption in obese and normal-weight men, glucagon concentration increased during activity and a recovery period of 4 hours thereafter. Some studies have shown that glucagon secretion increases with increasing catecholamines during exercise. An experimental study on male Wistar rats after 12 weeks of endurance training with different intensities on insulin and plasma glucose concentration was not showed significant difference between the concentration of insulin and glucose in the training group compared to the control group, although their concentration was lower in the training group. These results are consistent with the present study. Decreased blood glucose increases the glucagon hormone. In addition, increased amino acids, especially alanine and arginine, as well as exercise stimulate the secretion of this hormone (28).

\section{Conclusion}

Overall, the findings of the present study showed that eight weeks of HIIT or MICT with or without sodium alginate increased GLP-1, decreased glucagon, and improved anthropometric indices significantly. However, sodium alginate supplementation alone could not change the anthropometric indices, GLP-1, and glucagon. Therefore, HIIT or MICT alone or with sodium alginate supplement seem is a better approach to weight loss and reducing complications associated with overweight and obesity.

\section{Declarations}

Ethics approval and consent to participate: The present study was approved by the Ethics Committee of Razi University at Kermanshah-Iran. The informed consent form was filled out by all participants.

Availability of data and materials: All data is available

Competing interests: The authors declare that they have no competing interests.

Funding: There were no funding supports.

Consent for publication: Not applicable

Authors' contributions: R.A and M.A research design and data collecting and manuscript writing. W.T and H.A data analysis and manuscript writing. 
The authors would like to thank the subjects for their willing participation in this study.

\section{References}

1. Ghergherehchi R, Razaghi Azar M. Metabolic syndrome prevalence in obese children and adolescents. Medical Journal of Tabriz University of Medical Sciences. 2010;32(Suppl 3):57-61.

2. Kelly T, Yang W, Chen C-S, Reynolds K, He J. Global burden of obesityin 2005 and projections to 2030. International journal of obesity. 2008;32(9):1431-7.

3. Wasir J, Misra A, Vikram N, Pandey R, Gupta R. Comparison of definitions of the metabolic syndrome in adult Asian Indians. JAPI. 2008;56:158-64.

4. Boutcher SH. High-intensity intermittent exercise and fat loss. Journal of obesity. 2011;2011.

5. Wang N, Liu Y, Ma Y, Wen D. High-intensity interval versus moderate-intensity continuous training: Superior metabolic benefits in diet-induced obesity mice. Life sciences. 20.191:122-31;17

6. Williams BA, Grant LJ, Gidley MJ, Mikkelsen D. Gut fermentation of dietary fibres: physico-chemistry of plant cell walls and implications for health. International journal of molecular sciences. 2017;18(10):2203.

7. Mortazavi M, Seirafian S, Naini AE, Zamani N, Moien N. The Prevalence of Metabolic Syndrome in Patients on Hemodialysis and Peritoneal Dialysis: A Comparative Study. Journal of Isfahan Medical School. 2012;29.(171)

8. Yari Z, Mirmiran P, Moslehi N. Effects of Flaxseed and ItsNutritional Components on the Metabolic Syndrome and Associated Risk Factors. 2014.

9. Paxman J, Richardson J, Dettmar P, Corfe B. Daily ingestion of alginate reduces energy intake in free-living subjects. Appetite. 2008;51(3):713-9.

10. Ramos, J. S., Dalleck, L. C., Borrani, F., Beetham, K. S., Mielke, G. I., Dias, K. A., .. \& Coombes, J. S. (2017). Highintensity interval training and cardiac autonomic control in individuals with metabolic.

11. Matos VAF, de Souza DC, Browne RAV, dos Santos VOA, Costa EC, Fayh APT. Acute effect of high-intensity interval exercise and moderate-intensity continuous exercise on appetite in overweight/obese males: a pilot study. Sport Sciences for Health. 2017;13(2):403-10.

12. Nikroo H, Barancheshme M. The Comparison of Effects of Aerobic Interval and Continuous Training Program on Maximal Oxygen Consumption, Body Mass Index, and Body Fat Percentage in Officer Students. Journal of Military Medicine. 2014;15(4):245-51.

13. Soori R, Asad M, Barahouei-Jamar Z, Rezaeian N. The effect of aerobic training on the serum level of adipolin and insulin resistance in overweight men. KAUMS Journal (FEYZ). 2016;19(6):495-503.

14. Kurdi M, Ghorbanian B. Changes of body weight, insulin resistance, maximal oxygen uptake and some of physiological factors in partly obese adolescents in response to endurance training. journal of sports medicine and physical fitness. 2014;1(2):23-36. 
15. Farazmandi A, Rezaeian N. Effect of High Intensity Interval Training on Serum Levels of Progranulin and Insulin Resistance Index in Young Sedentary Overweight and Obese Women. Journal of Applied Exercise Physiology. 2020;16(31):20-1.

16. Gillen JB, Gibala MJ. Is high-intensity interval training a time-efficient exercise strategy to improve health and fitness? Applied physiology, nutrition, and metabolism. 2014;39(3):409-12.

17. Georg Jensen M, Pedersen C, Kristensen M, Frost G, Astrup A. efficacy of alginate supplementation in relation to appetite regulation and metabolic risk factors: evidence from animal and human studies. Obesity reviews.

2013;14(2):129-44.

18. Tabibirad S, Abednatanzi H, Nikbakht H, Ghazalian F, M. G. EFFECTS OF AEROBIC TRAINING ON DIPEPTIDYL PEPTIDASE-4 AND GLUCAGON-LIKE PEPTIDE-1 IN OBESE WOMEN WITH TYPE 2 DIABETES. Iranian Journal of Diabetes and Metabolism. 2019;18(5):275-81.

19. Hallworth JR, Copeland JL, Doan J, Hazell TJ. The effect of exercise intensity on total PYY and GLP-1 in healthy females: A pilot study. Journal of nutrition and metabolism. 2017 Feb 13;2017.

20. Martins C, Aschehoug I, Ludviksen M, Holst J, Finlayson G, Wisloff U, Morgan L, King N, Kulseng B. High-intensity interval training, appetite, and reward value of food in the obese. Medicine and science in sports and exercise. 2017;49(9):1851-8.

21. Islam H. The Effects of Exercise Intensity on Acylated Ghrelin, Active Glucagon-like Peptide-1, and Appetite: Examining The Potential Involvement of Interleukin-6.

22. Jafary A, Talebi Garakani E, Saghebjoo M, Fathi R. Effect of Aerobic Interval Training on Acylated Ghrelin, Peptide Tyrosine Tyrosine and Glucagon-Like Peptide-1 in Healthy Young Men. Sport Physiol 2019;11(41):17-34.

23. Lee SS, Yoo JH, So YS. Effect of the low-versus high-intensity exercise training on endoplasmic reticulum stress and GLP-1 in adolescents with type 2 diabetes mellitus. Journal of physical therapy science. 2015;27(10):3063-8.

24. Farzanegi P. The effects of aerobic training and arbutin on GLP1 and GLP1R in diabetes Rats. Indian J Fundam Appl Life Sci. 2014; 4:2231-6345.

25. Abdolmaleki A, Samavatisharif M, Nikbakht-Nasrabadi P, Amini R. The Effects of 12 Weeks of Low-volume Highintensity Interval Training and Traditional Continuous Exercise Training on Adiponectin Level and Lipids Profile in Obese Young Men. Scientific Journal of Ilam University of Medical Sciences. 2014;22(5):150-9.

26. Ueda S-y, Yoshikawa T, Katsura Y, Usui T, Nakao H, Fujimoto S. Changes in gut hormone levels and negative energy balance during aerobic exercise in obese young males. Journal of Endocrinology. 2009;201(1):151.

27. Fathi R, Ghanbari-Niaki A, Kraemer RR, Talebi-Garakani E, Saghebjoo M. The effect of exercise intensity on plasma and tissue acyl ghrelin concentrations in fasted rats. Regulatory peptides. 2010;165(2-3):133-7.

28. Gyntelberg F, Rennie MJ, Hickson RC, Holloszy JO. Effect of training on the response of plasma glucagon to exercise. Journal of Applied Physiology. 1977 Aug 1;43(2):302-5. 